\title{
Recent progress in physical sensing with optical microfiber technology
}

\author{
George Y. Chen \\ Optoelectronics Research Centre, University of Southampton, Southampton, SO17 1BJ, UK \\ gyc1g09@orc.soton.ac.uk
}

\begin{abstract}
An overview of selected developments in the field of optical microfiber physical sensors is presented, highlighting the key issues underpinning recent research and illustrating a number of important applications.

OCIS codes: $010.0280,060.2280,060.2370,060.5060,120.3180,120.4610,120.5410,120.6780,120.6810,230.2240$, $230.3990,230.4555,230.5750,280.4788,280.6780$
\end{abstract}

\section{Introduction:}

The technology and applications of optical fibers have progressed very rapidly in recent years. Fiber-optic sensors have been commercially successful and well established in various industries from biomedical to defense. They exhibit many advantages over their electrical counterparts, including higher responsivity, higher detection bandwidth, higher temperature performance, better immunity to electromagnetic interference, all-dielectric composition, greater environmental ruggedness and distributed sensing capability. However, the physical dimensions and the minimum bend radius of the optical fiber sets a lower limit on the final package size. In applications where the working space is stringent or where physical intrusion must be minimized, it becomes highly desirable to develop ultra-compact sensors that can maintain the level of performance despite the miniaturization.

The recent emergence of optical microfibers (OM) has opened up a new era of technological innovations. OMs confine light due a refractive index difference between the solid core and the external medium cladding. The fraction of light that propagates in the cladding depends on the ratio of the fiber diameter to the wavelength of light. OMs have the potential to solve the problem with its range of enabling properties, including large evanescent field, strong optical confinement, bend insensitivity, low stiffness and high configurability.

By significantly reducing the size of sensors and their associated electronics with supporting hardware, miniaturization offers the possibility of complete portable micro-systems which can carry out many of the operations traditionally performed in a laboratory. The other benefits of high compactness due to bend insensitivity include easier deployment and minimal intrusion. The consequential reduction in weight also draws substantial interest, particularly from the aerospace industry. It is widely recognized that for sensors responding to a mechanical stimulus, the better sensitivity associated with the higher responsivity resulting from the lower stiffness of OMs grants a notable enhancement in the detection of weak signals of the measurand. As for current sensors based on the Faraday Effect, the higher detection bandwidth hosted by the shorter optical path length resulting from the bend insensitivity of microfiber coils (MC) enables the measurement of faster-changing current signals.

This paper covers the innovative development $[1,2]$ of relatively unexplored areas of OM-based sensing as well as the envisioning of performance-enhancing techniques that can shape the on-going development of such sensors. In particular, extensive advancement was made in light of the simple demonstration of a novel current sensor with potentially gigahertz detection bandwidth. This includes the development of the resonator design to achieve higher compactness, and the first reported fabrication of the spun microfiber (SOM) to counter the effects of linear birefringence. Well-established and successfully proven sensing configurations such as the flexural disc (FD) and air-backed mandrel (ABM) were adopted to create miniaturized OM-based accelerometers and microphones, with potential responsivity enhancements of at least one order of magnitude.

\section{Temperature sensing}

The preliminary exploration involved the development of a compact thermometer capable of sliding along electrical wires to detect electrical arcing-induced hot spots. This was devised using a microfiber coil resonator (MCR) coiled around a Teflon tube, as shown in Fig. 1 (a). The magnitude of the temperature-induced resonant wavelength shift can be tailored by the choice of materials, due to thermal expansion and thermo-optic effects. For the first time, MCRs were designed with such mobility so they can efficiently measure the temperature profile of a rod-like structure. This type of sensor can be potentially used in industrial applications to inspect insulated wires and cables for insulation defects, aiding the prevention of electrical fires. 

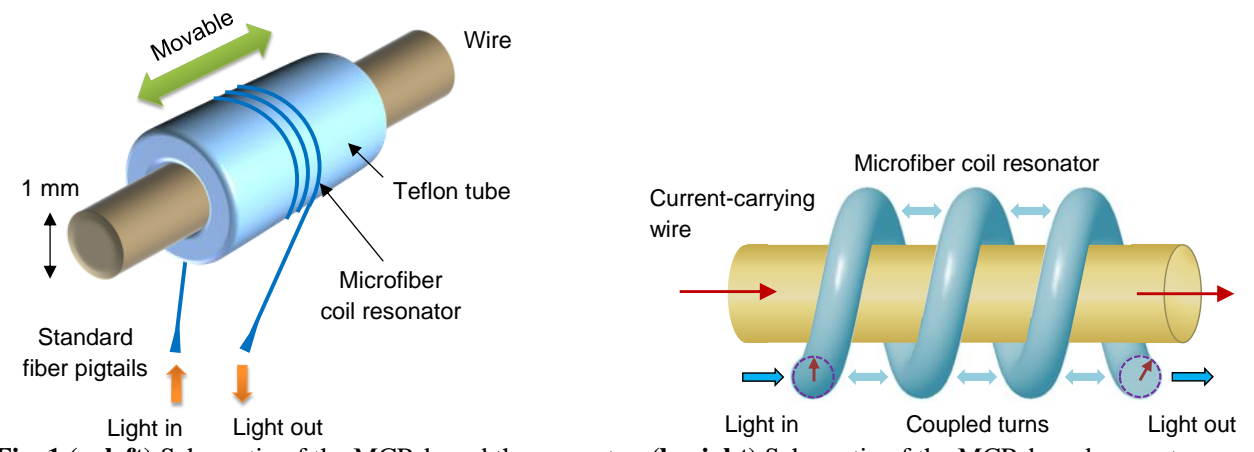

Fig. 1 (a, left) Schematic of the MCR-based thermometer. (b, right) Schematic of the MCR-based current sensor.

\section{Current sensing}

The same physical arrangement of self-coupled OM coiled around a wire was subsequently established as a current sensor. Current-induced variations in the local magnetic field axial to the direction of light rotate the polarization azimuth via the Faraday Effect. The relatively short length of OMs allows short transit times of light, giving rise to potentially gigahertz detection bandwidth for fast current detection. The responsivity to current was linearly proportional to the number of OM turns.

A means to increase the current responsivity without being limited by the maximum producible OM length was investigated. It was subsequently demonstrated both theoretically and experimentally that resonantly enhanced Faraday rotation can be realized in MCRs, and it is a function of the extinction ratio. However, by increasing the optical path length there is an unavoidable trade-off between the responsivity and the detection bandwidth.

The upmost-compact resonator configuration shown in Fig. 1(b) is achieved at the expense of temporal and wavelength instability. Thermal drifts and vibrations transfer from the ambient environment can modify the geometrical and optical properties of the MCR, thereby radically detuning the operating wavelength from the resonant condition and thus altering the Faraday rotation. This issue was solved using two approaches, one being passive and the other active. The first approach (Fig. 2(a)) involved optimizing the spatial arrangement of the OM turns with refractive index chirping to instigate a broadening of the lower region of the resonance shape such that its output transmittance is much more tolerant to resonant wavelength fluctuations. The second approach (Fig. 2(b)) incorporated a piezoelectric element inside the MCR package as a support rod, which can be actively tuned using feedback control to lock the resonance detuning.

Linear birefringence associated with packaged MCs and MCRs was another major concern. This characteristic is typical of devices operating with the Faraday Effect. Bend- and packaging-induced birefringence causes the differential phase between the eigenmodes in the two orthogonal axes to increase with optical path length, resulting in alternating polarities of Faraday rotation that produce zero net rotation at half beat length intervals. The problem was solved by introducing a twist during the fabrication of highly birefringent OMs to create the first reported SOMs. The magnitude of the intrinsic linear and circular birefringence can be optimized to deliver a good balance between resistance to external effects and responsivity.

Although SOMs are capable of delivering near-maximum performance for non-resonator sensor heads, resonantly enhanced OM devices would require further design optimizations due to their structural complexity. The exploration of OM resonator designs led to the investigation of microfiber loop resonators (MLR). It was found that design optimizations are possible to host efficient Faraday rotation for both birefringent and non-birefringent sensor heads. This study provided the groundwork for designing practical current sensors using MLR sensor heads with a high responsivity to current.
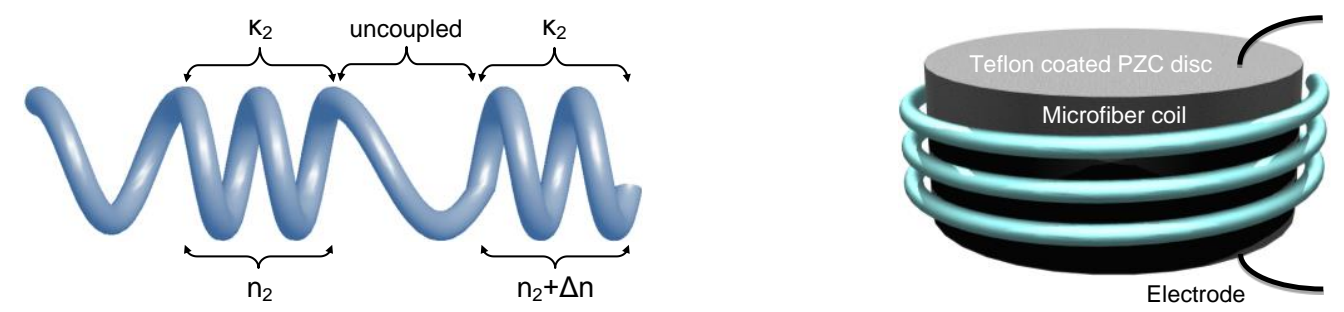

Fig. 2 (a, left) Schematic of the chirped MCR. (b, right) Schematic of the piezoelectric disc coiled with MCR. 
Going back to non-resonator sensor heads, an alternative to the SOM was proposed in the form of a novel postfabrication technique for reversing the detrimental effect of linear birefringence in MC-based current sensors. By means of changing the local birefringence at the right positions along the fiber axis, the differential phase of light can be progressively optimized to deliver uni-directional Faraday rotation and thus maximum responsivity.

\section{Acceleration sensing}

Diverging to the exploration of other sensing applications, a compact accelerometer shown in Fig. 3(a) based on a centrally supported FD overlaid with OM was modeled and demonstrated. Both the acceleration responsivity and detection bandwidth were influenced by the choice of disc material and geometry. There are several benefits from down-scaling the traditional accelerometer, including easier deployment, less intrusive and lower cost. Due to the large minimum bend radii of standard optical fibers, to achieve such small package sizes would result in severe polarization-dependent loss and depolarization. The excellent bend insensitivity of OMs enables very compact devices to be made without compromising their performance. Moreover, the responsivity scales with the length of $\mathrm{OM}$ and it can be potentially several orders of magnitude higher than conventional designs using standard optical fibers.
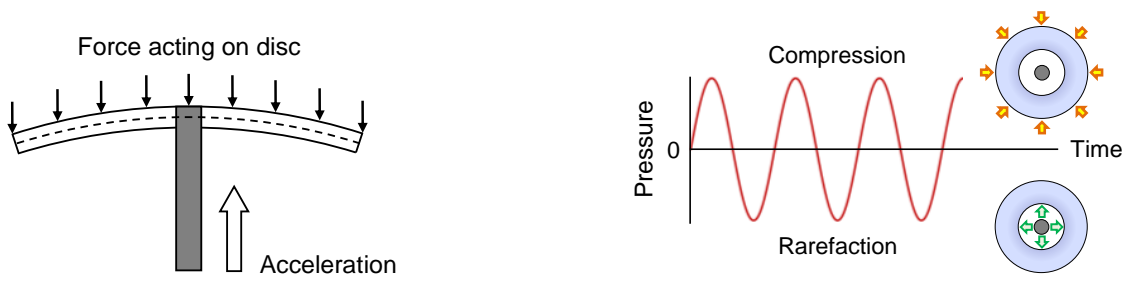

Fig. 3 (a, left) Illustration of the OM-based FD accelerometer. (b, right) Illustration of the MC-based ABM microphone.

\section{Acoustic sensing}

For a different purpose, a compact microphone shown in Fig. 3(b) based on an ABM coiled with OM was proposed and demonstrated. Both the acoustic responsivity and detection bandwidth were dependent on the material and geometry of the mandrel. Likewise, there are benefits with the compactness of the device, notably for defence applications. The microphone showed the potential for very high responsivity despite the miniaturization, which generates strong interest for future development. Another possible direction is the development of a hydrophone for underwater acoustic sensing.

\section{Summary}

The results achieved so far represent a significant advance in harnessing OM technology for sensing. It would be useful to combine the numerous techniques developed so far when engineering a sensing system to attain the best possible performance. Aside from achieving unprecedented high compactness, MC/MCR sensor heads have the potential to raise the detection bandwidth of traditional fiber-optic current sensors by at least one order of magnitude. Accelerometers and microphones employing OMs can expect a similar leap of improvement in their responsivity, which gives rise to enhanced sensitivity. Although the experiments were performed with relatively short OM lengths and a host of further scientific and more practical challenges remain, these results can be considered an important step towards demonstrating the feasibility of using OMs for sensing. While still in their infancy, these sensors promise a tremendous impact on a wide range of technological applications in industries such as biomedical, defence, navigation, oil \& gas, process control and transportation. One should expect substantial activity in the rise of OM technology in the coming decade, and significant progress should be anticipated in the development of OM-based sensors as well as their outlook to establishing commercial interest.

\section{References}

[1] G. Y. Chen, M. Ding, T. P. Newson, and G. Brambilla, “A review of micro- and nanofiber based optical sensors”, Open Opt. J. 7, 32-57 (2014).

[2] G. Y. Chen, T. P. Newson, and G. Brambilla, “Optical microfibers for fast current sensing”, Opt. Fiber Technol. 19, $802-807$ (2013). 